\title{
Educación indígena: Una educación propia del pueblo Nomatsigenga
}

\section{Indigenous education: A nomatsigenga people own education}

\author{
Jaime Shoente Chumpate ${ }^{1}$ \\ Programa Descentralizado de Formación de Profesores en EIв Nivel Primaria \\ Universidad Nacional Mayor de San Marcos \\ jaimeshoo8_@hotmail.com
}

\begin{abstract}
Resumen
En este artículo presento una propuesta de una educación indígena propia del pueblo Nomatsigenga a partir de una experiencia educativa en el aula, en cuatro escuelas nomatsigenga de tipo unidocente y polidocente donde se aplica la Educación Intercultural Bilingüe. Consiste en asegurar la participación activa de los sabios nomatsigenga en todo el proceso escolar, desde el diagnóstico sociocultural, pasando por la elaboración del diseño curricular de la escuela hasta el proceso didáctico en el aula, conjuntamente con los docentes de Еıв nombrados por el Estado.
\end{abstract}

Palabras claves: Educación Intercultural Bilingüe, unidocente, educación indígena, selva central, nomatsigenga, cultura, pueblo y cosmovisión.

\begin{abstract}
A proposal of own indigenous education of the Nomatsigenga people is presented in this article. It is based on an educational experience in the classroom, in four Nomatsigenga schools of a unique-teacher and multi-teacher type where Intercultural Bilingual Education (IBE) is applied. We propose guaranteeing the active participation of the Nomatsigenga wise people in the entire school process. It means that these wisepeople will express their points of view and will decide about the sociocultural diagnosis, the elaboration of the school's curricular design and also the didactic process in the classroom, from peer to peer with the IBE teachers appointed by the State.
\end{abstract}

Keywords: Intercultural Bilingual Education, unique-teacher, indigenous education, central jungle, Nomatsigenga, culture, indigenous people and cosmovision.

$1 \quad$ Estudió Nivel Primario en la I.E.B. N 30672 Alto Anapati, cursó Nivel Secundario en la I.E.I.P.P. Aldea del Niño Mazamari, elaborador de cuaderno de trabajo en lengua Nomatsigenga comunicación Majari $4^{\circ}$ y $6^{\circ}$. Actualmente es docente en la Instituciones Educativas Bilingües de la UGEL PANGOA- SATIPO- JUNIN. 


\section{Jaime Shoente Chumpate}

\section{Introducción}

Tras cuatro años de experiencia educativa en el aula, en cuatro escuelas nomatsigenga de tipo unidocente y polidocente, concebimos que la Educación Intercultual Bilingüe es útil para analizar, aplicar y mejorar la educación de nuestros estudiantes. Por ello, hemos escrito este artículo pensando tanto en profesores en ejercicio como en aquellos en formación, pero esperamos que sea de interés para cualquier persona involucrada directa o indirectamente en el replanteamiento crítico de la educación. Lo que tratamos de ofrecer al lector es, fundamentalmente, un desafío para replantear y reformular sus ideas y prácticas educativas, proporcionándole un contexto desde el que sea posible. Se trata de un replanteamiento crítico de las estrategias de los procesos de enseñanza y aprendizaje, para decidir si se han de cambiar y, de ser así, lo que ha de cambiarse.

En consecuencia, los docentes de cualquier lengua y cultura debemos asumir el compromiso de mejorar el nivel educativo de nuestros estudiantes para que sean ciudadanos competentes ante cualquier situación. Además, hay que tener en cuenta que nuestro país es pluricultural y multilingüe, donde coexisten 47 lenguas originarias (Ministerio de Educación del Perú, 2015). De esta diversidad debemos partir en nuestro trabajo pedagógico dentro del aula, ya que existenun sustento científico que avale la idea de que una cultura es superior a las otras.

No hubiéramos escrito este artículo si no fuera por la influencia de nuestros colegas de la Red Educativa INTATONI, quienes desde hace muchos años intentan cambiar la forma de educar a los niños nomatsigenga. A ellos les agradecemos, como también al gran pueblo nomatsigenga, en especial a los niños y niñas de Mayni, Tsonkireni, Alto Anapati y Chuquibambilla, con quieres mantuvimos diálogo y compartimos costumbres. Asimismo, agradecemos a nuestras familias que día a día comprenden nuestra ausencia en el hogar; a nuestros hijos, Jaime Gabriel y Miyashiro Sebastián, que son la fortaleza y el motor de nuestras acciones; a su madre, que nos acompañó durante un tiempo de nuestro trabajo; a nuestros padres - Jorge y Martha, portadores de conocimientos de nuestros pueblos-, a cuya memoria dedicamos este trabajo; $y$, por último, a nuestros compañeros y maestros de la Universidad Nacional Mayor de San Marcos, en especial a Jairo Valqui, Lucy Trapnell, Gonzalo Espino, Evelyn Chauca, Antonio Sancho, Alicia Alonso, Fermín Tiwi, Juan Loayza, Manuel Conde, Rosario Basurto y a la recordada dra. María Cortez Mondragón, quien nos acompañó desde el inicio de formación profesional. 


\section{Declaración de principios ${ }^{2}$}

Los autores de este artículo compartimos una filosofía próxima a la educación inclusiva para todos en las escuelas bilingües nomatsigenga. El hecho de que la escuela sea para todos, implica que el profesor tiene el deber de atender de forma individual las necesidades de todos sus estudiantes, en el contexto de una escuela inclusiva, a donde todos los estudiantes pertenezcan por igual y disfruten de los mismos derechos. Es decir, si en una institución educativa nomatsigenga $\mathrm{X}$, de tipo unidocente, se encuentran dos estudiantes de habla asháninca y uno de habla castellano, el profesor debe tener muy en cuenta el «tratamiento de la lengua» y la enseñanza personalizada, para que cada uno de ellos logre entender con claridad las enseñanzas. Por tanto. el docente debe ser conocedor y mediador de las culturas de sus estudiantes.

En nuestra visita a las escuelas de las comunidades de Mayni y Chuquibambilla, en el 2013 y 2017 respectivamente, escuchamos opiniones de los padres de familia tales como «la enseñanza en nomatsigenga es un atraso», «ya no debemos hablar nuestra lengua, ¿para qué?», «ya no queremos que los profesores bilingües lo enseñe a nuestros hijos». Sin embargo, al pedirles que justifiquen el porqué, no encontramos respuesta que fundamente dichas opiniones. Estas ideas son insufladas en la población por aquellos que están en contra del bilingüísmo, quienes sostienen que no es necesario que la lengua originaria se desarrolle en sesiones de clases, puesto que ya se domina y se debe aprender una lengua «superior» como es el castellano. Mas nosotros tenemos muy clara y defendemos la idea de que no hay una lengua que sea mejor o superior a las otras. Los pobladores de aquellas comunidades comprendieron que todas las personas empleamos prejuicios y estereotipos cuando nos relacionamos con los demás, y que para liberarnos de esas taras, es necesario conocer otras sociedades diferentes a la propia.

\section{Lo que la Educación Intercultural Bilingüe es y no es.}

La educación se considera en nuestros días como un derecho «natural» $\mathrm{y}$ «universal». Esta consideración se relaciona con dos conceptos: la infancia, como periodo vital específico cuyo principal objetivo es preparar a las personas para su

2 Temas consensuados en la escuela de Mayni. 
«vida futura» por medio de la educación; y el progreso, como un ideal de desarrollo humano directamente vinculado al bienestar social y a la felicidad individual.

Visto así, se entiende la infancia como una especie de "pre-vida», y quizá es ésta una de las razones que hacen que la escuela parezca a menudo un lugar muy artificial y alejado de la «vida real» de un nomatsigenga. Sin embargo, el término «infancia» no es en modo alguno unívoco ni universal: pensarlo como tal no es más que un intento de ocultar las diferentes circunstancias y situaciones sociales en las que viven los niños y los jóvenes.

Por otra parte, el concepto moderno de progreso se relaciona con el constante aumento de la producción y el consumo, la economía de tiempo, la maximización de la eficacia y el beneficio, y el predominio de la actividad económica, olvidando sus efectos negativos sobre la calidad de vida y el desarrollo de los seres humanos.

Por el contrario, la Educación Intercultural Bilingüe, por un lado, compete a todas las decisiones que tienen que ver con la educación, y, por otro, reclama un cambio en la concepción de las relaciones sociales y en otras variables relevantes en educación. Su efectividad depende, en gran medida, de los requisitos individuales y grupales en los contextos específicos en los que se pone en práctica, pero también de la posibilidad de que se combine con medidas estructurales que van más allá del entorno educativo.

De no cumplirse estas condiciones, todas las iniciativas que parecen interculturales pueden devenir en recurso de desigualdad, envitando que se ponga en práctica iniciativas genuinas de respeto a la diversidad cultural para seguir celebrando superficialmente las diferencias desde una perspectiva «turística». Y la Educación Intercultural Bilingüe no es celebrar aisladamente las diferencias, como por ejemplo, las llamadas interculturales, días gastronómicos, día de la paz, etc.

\section{Propuesta para desarrollar la Educación Intercultural Bilingüe en la Amazonia Peruana, Selva Central}

Antes de comenzar con el análisis de datos, es necesario indicar que el investigador de este estudio fue el coordinador del proyecto a la vez que el diseñador del modelo de implementación de la Educación Intercultural Bilingüe que promueve esta iniciativa. Debido a su formación como docente y a su identidad nomatsigenga, el autor se siente profundamente comprometido con el desarrollo de esta investigación, en la que esbozará, desde su punto de vista, los roles que deben 
cumplir todos los actores, ejecutores y partícipes de la implementación de este enfoque.

La participación de estos actores es importante ya que el enfoque intercultural alude a una participación simétrica de todas las culturas, las que en este caso están representadas por los Educadores Bilingües Nomatsigenga. Por lo tanto, en este estudio recogemos todas las opiniones y las interpretamos desde nuestro punto de vista, que tiene como premisa que la construcción del conocimiento no sólo se caracteriza por ser colectiva, sino que, además, debe ser democrática y pluralista por lo que pueden participar todas las formas de pensar.

Iniciaremos este análisis con la información recabada a través de la observación, para lo que se aplicaron dos pautas ya explicadas en el capítulo que habla de la metodología de estudio.

Desde hace ya varias décadas, en el Perú se viene generando un fenómeno social que ha cambiado la imagen del país: la elevación de las ciudades como espacios que propician la vida exitosa y las oportunidades para todos. A ello se suman los problemas y conflictos de distribución de tierras u otros recursos naturales que han provocado la segregación de muchos grupos humanos, sobre todo de aquellos que son vistos por la sociedad como inferiores, aquellos que son conquistados, colonizados y dominados. De a pocos, estos grupos se vieron forzados a abandonar sus espacios que no solo eran de su propiedad sino también de su administración, desplazándose a las grandes urbes en busca de una vida mejor y moderna. Este fenómeno, que no es exclusivo del Perú, ha impuesto nuevas formas de agruparnos en el mundo, de pensar nuestros roles y, sobre todo, de concebir nuevas formas de vida.

Los pueblos indígenas han sido los más afectados a nivel nacional por este proceso de distribución de la tierra y, en consecuencia, han ido migrando hacia los grandes centros urbanos ubicados en la zona central del país en un proceso constante desde hace mucho tiempo. Es Lima, la capital, la que recibe día a día el mayor número de migrantes, a los que en los últimos años se han venido sumando los inmigrantes de otros países de América Latina. Pero en comunidades como Alto Anapati, que se encuentra a más de cien kilómetros de Lima, también se han ido desplazándose los hijos de los indígenas nomatsigenga, los que a la vez han formado familias cuyos hijos e hijas se educan en escuelas preferentemente de Educación Intercultural Bilingüe.

Es por esta razón que desarrollamos este estudio, el cual tiene como propósito diagnosticar la propuesta de Educación Intercultural Bilingüe en las instituciones 


\section{Jaime Shoente Chumpate}

educativas focalizadas por los Asistentes de Soporte Pedagógico Intercultural (ASPI), en cuanto a su implementación y a la propuesta didáctica diseñada para ello. Esta propuesta forma parte del convenio establecido entre la Organización de Pueblos Indígenas del Valle de Pangoa (KANUJA), y la Organización de Maestros Bilingües Nomatsigenga y Ashaninka de Pangoa (omabinoaP), durante el año 2016, cuyo proyecto se denominó, «Visiones en Torno a la Didáctica de la Educación Intercultural Bilingüe en la Comunidad Nativa de Alto Anapati, Estudio de Caso: Implementación de Educación Intercultural Bilingüe en las Instituciones Educativas Focalizadas».

A través de este enfoque educativo, nuestro estudio intenta poder cumplir con la añorada «educación de calidad» para aquellas generaciones que forman parte de esta comunidad y más aún como una propuesta para todo nuestro país, caracterizada por su diversidad multicultural y multiétnica.

La metodología didáctica de esta propuesta consiste en la realización de un diagnóstico sociocultural de la comunidad, para conocer las actividades sociales y productivas del contexto, lo que permitirá que sean plasmados en la Programación Anual Curricular (PCA). Estas actividades descritas nos permitirán realizar el Proyecto de Aprendizaje Intercultural (PAI) de cada mes. De esta manera se facilitará el aprendizaje oportuno en los estudiantes.

El Proyecto de Aprendizaje Intercultural es una unidad didáctica innovadora para lo cual se creó una matriz con el apoyo de los Asistentes de Soporte Pedagógico Intercultural (ASPI), que pudiese desarrollar efectivamente los procesos necesarios que se evidencia en el diagnóstico psicolingüístico y sociocultural de una población dada. Por ejemplo, en Mayni, una escuela bilingüe nomatsigenga, el nivel de dominio de la lengua está en el «ESCENARIO 1», que consiste en que la población en general domina y habla al 100 \% la lengua materna y muy poco conoce el castellano. Asimismo, en el mes de marzo la población se dedica a la cosecha del café, una actividad prioritaria debido a su potencial pedagógico y cultural. De aquí nace el Proyecto de Aprendizaje Intercultural, que para el contexto se denominó «Nos integramos en la cosecha de café». De esta manera, los temas de los proyectos se eligen de acuerdo al calendario comunal.

Los Proyectos de Aprendizajes Interculturales se diseñaron considerando la propuesta didáctica entregada en la Planificación Curricular Anual donde están insertadas las actividades del calendario comunal, que es el objeto en estudio; y si estas son coherentes y pertinentes en su contenido, a la vez es muy importante demostrar la participación de los dos actores involucrados en este diseño que son el

60 Lengua y Sociedad 
docente de aula y el sabio nomatsigenga. También se entrega información acerca de las formas de inclusión de los conocimientos de las diversas culturas indígenas en este proyecto y evidencia la intencionalidad del trabajo realizado en aula, demostrando las formas de relación y jerarquía existentes entre el Nomatsigenga (sabio Indígena), el docente y los estudiantes (la participación del sabio nomatsigenga se debe realizarse en un contexto real de los nomatsigenga, mas no en la escuela).

\section{Conclusión}

Concluir este artículo ha sido complejo, ya que la experiencia que se realizó en cuatro escuelas de la Amazonia Peruana (Mayni, Tsonkireni, Alto Anapati y Chuquibambilla) en cuanto a su implementación y a la propuesta didáctica, contemplando la participación del sabio o la sabia indígena al interior de la sala de clases y agregando una propuesta de diversificación curricular, es un caso innovador ya que se realiza por primera vez y es necesario elegir otro espacio de aprendizaje (campo, río, espacio deportivo, parque y/o casa comunal) de acuerdo a la planificación del docente a cargo. Visualizar como se imaginan los actores que participan en esta experiencia en torno a cómo debe ser la Educación Intercultural Bilingüe en este espacio, donde la población nomatsigenga es minoritaria pero de calidad y donde los pocos estudios académicos realizados con anterioridad no desarrollan una temática vinculada a esta investigación, conlleva una dificultad acerca de cómo plantearse o desde donde hacerlo para evaluar lo recopilado como una contribución a la implementación de este enfoque.

Se ha planteado entonces todo lo observado y recopilado como un aporte a esta visión, teniendo como fundamento las definiciones de este paradigma de la interculturalidad y la importancia del bilingüismo

La acepción social de la iniciativa en el ámbito local y la toma de conciencia por parte de los distintos docentes sobre la coexistencia actual de diversas culturas indígenas en un mismo espacio territorial, es uno de los más importantes logros obtenidos, pues permite desarrollar un compromiso con los gestores de futuras iniciativas como esta. Todo lo anterior se consiguió debido al modelo de gestión pedagógica que adoptó el proyecto en esta oportunidad, decidiéndose en esta oportunidad dar un rol más importante a los Sabios Nomatsigenga, los que debieron tomar decisiones y adecuar las jornadas de educación tradicional de 


\section{Jaime Shoente Chumpate}

acuerdo a la planificación de Proyecto de Aprendizaje, que diseñaron en conjunto con los docentes.

Los Sabios nomatsigenga entonces son considerados sólo como una especie de recursos didácticos, con la misma orientación y función de una biblioteca de aula, que contribuyen a completar los conocimientos que el docente requiere para desarrollar su labor. No se desarrolla una interacción equitativa entre ambos actores para fortalecer la creación de los Proyectos de Aprendizajes Interculturales, que son los proyectos pedagógicos de aula que permitirán generar ambientes interculturales en contextos multiculturales.

Los resultados expuestos en párrafo anterior, manifiestan el conflicto en la interacción que desarrolla el docente con Sabio Indígena. Este conflicto marcado por la ausencia del diálogo en condiciones de igualdad, el que debe desarrollarse en un «encuentro auténtico con el otro que crea vínculos de reconocimiento, respeto, solidaridad y amistad. Se deja se usar instrumentalmente al otro o de considerarlo como un objeto. Se instaura la modalidad dialógica de la interlocución, en la que los participantes por igual se enriquecen y transforman» (Godenzzi, 2005). Para lo que en contextos urbanos y multiculturales se debe convocar a todos a desconstruir aquellas conductas discriminatorias que sitúan al otro en situación subalterna, esta preparación requiere de reflexión, voluntad y transformación de la visión que se tiene de las comunidades nomatsigengas.

A partir del discurso recuperado de los docentes y sabios nomatsigengas acerca de cómo se incluyen otras culturas en el aula, se rescataron una serie de ideas para implementar mejor el enfoque intercultural en el aula. Estas ideas están planteadas desde la mirada de los profesores y sabios nomstigenga que participaron como actores directos en el proyecto en que se basa este artículo. Estas propuestas aparecen a continuación:

- Generar un programa de capacitación sobre culturas indígenas que sea desarrollada por los Sabios Indígenas e incluya la particular visión de los indígenas urbanos. Lo que contribuiría a mejorar la valorización de los educadores indígenas por parte de los docentes y a generar el trabajo colaborativo entre ambos actores.

- Entregar una capacitación continua a cargo de un especialista en Educación Intercultural Bilingüe, con dedicación absoluta, sobre todo durante el «Mes de la Interculturalidad», que contemple temas como teorías del aprendizaje,

62 Lengua y Sociedad 
currículo y didáctica en el nivel de educación inicial y educación primaria para la interculturalidad.

- Diseñar y planificar el trabajo desde Marzo e incluir los contenidos indígenas de manera transversal en la organización del currículo para la planificación de la enseñanza considerando todos los sectores y/o ámbitos presentes en las bases curriculares de los niveles de educación inicial y primaria.

Gestionar la posibilidad de adquirir mayores recursos didácticos para la Educación Intercultural Bilingüe sobre todo en lo que respecta a las lenguas indígenas, para el nivel de educación primaria. Esto permitirá generar una propuesta didáctica para la enseñanza de las lenguas indígenas como segunda lengua en las zonas donde poco se habla la lengua nomatsigenga.

\section{Referencias Bibliográficas}

Aikman, S. (2003). La educación indígena en Sudamérica. Interculturalidad y Bilingüismo en Madre de Dios. Lima: Instituto de Estudios Peruanos.

Calero, M. (2002). ¿Nuevo enfoque Educativo? Realidades e Idealidades. Lima: San Marcos.

Guevara, G. (2014). Introducción a la Teoría de la Educación. Lima: Trillas-UAM. Ministerio de Educación del Perú. (2015). Rutas del aprendizaje. ¿Qué y cómo aprenden nuestros estudiantes? V ciclo. Área Curricular: Comunicación. $5 .^{\circ}$ y 6. ${ }^{\circ}$ grados de educación primaria. Lima: Autor.

Ministerio de Educacion del Perú. (2014). Komantagantsipage Majari, ogotaige OMAGARO. Lima: CECOSAMI.

Pancorbo, V. (2008). Educación Intercultural Bilingüe. Estrategias de Lectura en la Pedagogía Interactiva Inclusiva. Lima.

Trapnell, L. (2008). Interculturalidad, Conocimiento y Poder. Alcances de un proceso de investigación-acción en dos escuelas de la Amazonia Peruana. Lima: Instituto del Bien Común.

Vilchez, E. Valdez, S., \& Rosales, M. (Eds.). (2007). Interculturalidad y Bilingüismo en la Formación de Recursos Humanos: Educación, Medicina, Derecho y Etnodesarrollo. Lima: Instituto de Lingüística Aplicada cila / Universidad Nacional Mayor de San Marcos. 
Zuñiga, M., Pozzi, I., \& López, L. E. (1991). Educación Bilingüe Intercultural, Reflexiones y Desafíos. Lima: FomCIENCIAs. 